\title{
Monte Carlo Algorithm for Maneuvering Target Tracking and Classification ${ }^{\star}$
}

\author{
Donka Angelova $^{1}$, Lyudmila Mihaylova ${ }^{2}$, and Tzvetan Semerdjiev ${ }^{1}$ \\ 1 Institute for Parallel Processing, Bulgarian Academy of Sciences \\ 25A Acad. G. Bonchev St, 1113 Sofia, Bulgaria \\ donka@bas.bg \\ 2 Department of Electrical and Electronic Engineering, University of Bristol, \\ Merchant Venturers Building, Woodland Road, Bristol BS8 1UB, UK \\ mila.mihaylova@bristol.ac.uk
}

\begin{abstract}
This paper considers the problem of joint maneuvering target tracking and classification. Based on the recently proposed particle filtering approach, a multiple model particle filter is designed for two-class identification of air targets: commercial and military aircraft. The classification task is implemented by processing radar (kinematic) measurements only, no class (feature) measurements are used. A speed likelihood function for each class is defined using a priori information about speed constraints. Class-dependent speed likelihoods are calculated through the state estimates of each class-dependent tracker. They are combined with the kinematic measurement likelihoods in order to improve the process of classification. The performance of the suggested multiple model particle filter is evaluated by Monte Carlo simulations.
\end{abstract}

\section{Introduction}

A lot of research has been performed recently concerning the problem of joint target tracking and classification. Actually, the simultaneous implementation of these two important tasks in the surveillance systems facilitates the situation assessment, resource allocation and decision-making [1,2]. Classification (or identification) usually includes target allegiance determination and/or target profile assessment such as vehicle, ship or aircraft type. Target class information could be obtained from an electronic support measure (ESM) sensor, friend-and-foe identification system, high resolution radar or other identity sensors. It could be inferred from a tracker, using kinematic measurements only or in a combination with identity sensors. On the other hand, target type knowledge applied to the tracker can improve tracking performance by the possibility of selecting appropriate target models. Classification information can assist in correct data association and false tracks elimination in multiple target tracking systems.

Two basic alternative approaches to classification exist based on Bayesian and DempsterShafer theories. Comparative studies [1] of these inferring techniques come to the conclusion that Dempster-Shafer is more robust than the Bayesian technique, but this is

\footnotetext{
* Research supported in part by Center of Excellence BIS21 grant ICA1-2000-70016, by the Bulgarian Foundation for Scientific Investigations under grants I-1202/02 and I-1205/02, and in part by the UK MOD Data and Information Fusion Defence Technology Center.
} 
achieved at the expense of delay in time [2]. The Bayesian approach is more certain regarding the correct decisions. Efficient approximations to the optimal Bayesian solution of joint tracking and classification (JTC) are provided by Monte Carlo methods. Feasible implementations of the JTC task via particle filtering are reported in [34]. A unified algorithm for JTC is suggested in [2] within the framework of the Bayesian theory. A bank of filters, covering the state and feature space are run in parallel with each filter matched to different target class. A robust particle filter is proposed as a concrete realization of the algorithm.

The accurate representation of joint state and class probability distributions is an important advantage of the particle filtering approach. The highly non-linear relationships between state and class measurements and non-Gaussian noise processes can be easily processed by the particle filter. In addition, flight envelope constraints, which are essential part of the JTC task, can be incorporated into the filtering algorithm in a natural and consistent way [5].

The objective of the present paper is to explore the capabilities of this particle filtering technique to track and classify a maneuvering target. Two air target classes are considered: commercial aircraft and military aircraft. A bank of two interacting multiple model class dependent particle filters is designed and implemented. The novelty of the paper relies also on accounting for two kinds of constraints : both on the acceleration and on the speed. Two speed likelihood functions are defined based on a priori information about speed constraints of each class. Such kind of constraints are used in other approaches for decision making (see e.g. [6]). At each filtering step, the estimated speed from each class dependent filter is used to calculate a class dependent speed likelihood. The speed likelihoods are combined with kinematic likelihoods in order to improve the process of classification.

The remaining part of the paper is organized as follows. Section 2 summarizes the Bayesian formulation of the JTC problem according to [2,4 7,8]. Section 3 presents the developed multiple model particle filter using both speed and acceleration constraints. Simulation results are given in Section 4, with conclusions generalized in Section 5.

\section{Problem Formulation}

Consider the following model of a discrete-time jump Markov system, describing the target dynamics and sensor measurement

$$
\begin{aligned}
x_{k} & =F\left(m_{k}\right) x_{k-1}+G\left(m_{k}\right) u_{k}+B\left(m_{k}\right) w_{k}, \\
z_{k} & =h\left(m_{k}, x_{k}\right)+D\left(m_{k}\right) v_{k}, k=1,2, \ldots,
\end{aligned}
$$

where $x_{k} \in \mathbb{R}^{n_{x}}$ is the base (continuous) state vector with transition matrix $F, z_{k} \in \mathbb{R}^{n_{z}}$ is the measurement vector with measurement function $h$, and $u_{k} \in \mathbb{U}$ is a known control input. The noises $w_{k}$ and $v_{k}$ are independent identically distributed (i.i.d.) Gaussian processes having characteristics $w_{k} \sim N(0, Q)$ and $v_{k} \sim N(0, R)$, respectively. All vectors and matrices are assumed of appropriate dimensions. The modal (discrete) state $m_{k} \in \mathbb{S} \triangleq\{1,2, \ldots, s\}$ is a time-homogeneous first-order Markov chain with transition probabilities $p_{i j} \triangleq \operatorname{Pr}\left\{m_{k}=j \mid m_{k-1}=i\right\},(i, j \in S)$ and initial probability distribution $P_{0}(i) \triangleq \operatorname{Pr}\left\{m_{0}=i\right\}$ for $i \in S$, such that $P_{0}(i) \geq 0$, and 
$\sum_{i=1}^{s} P_{0}(i)=1$. We assume that the target belongs to one of the $M$ classes $c \in C$ where $C=\left\{c_{1}, c_{2}, \ldots, c_{M}\right\}$ represents the set of the target classes. Generally, the number of the discrete states $s=s(c)$, the initial probability distribution $P_{0}^{c}(i)$ and the transition probability matrix $\left[p_{i j}\right]^{c}, i, j \in S$ are different for each target class.

The joint state and class is time varying with respect to the state and time invariant with respect to the class [2]. Let $\left\{Z^{k}, Y^{k}\right\}=\left\{z_{i}, y_{i}\right\}_{i=1}^{k}$ be the cumulative set of kinematic $\left(Z^{k}\right)$ and class (feature) measurements $\left(Y^{k}\right)$ up to time $k$.

The goal of the joint tracking and classification task is to estimate the state $x_{k}$ and the posterior classification probabilities $P\left(c \mid\left\{Z^{k}, Y^{k}\right\}\right), c \in C$ based on all available measurement information $\left\{Z^{k}, Y^{k}\right\}$. If we can construct the posterior joint state-class probability density function ( $p d f) p\left(x_{k}, c \mid\left\{Z^{k}, Y^{k}\right\}\right)$, then the posterior classification probabilities can be obtained by marginalisation over $x_{k}$ :

$$
P\left(c \mid\left\{Z^{k}, Y^{k}\right\}\right)=\int_{x_{k}} p\left(x_{k}, c \mid\left\{Z^{k}, Y^{k}\right\}\right) d x_{k} .
$$

Suppose that we know the posterior joint state-class pdf $p\left(x_{k-1}, c \mid\left\{Z^{k-1}, Y^{k-1}\right\}\right)$ at time instant $k-1$. According to the Bayesian philosophy, $p\left(x_{k}, c \mid\left\{Z^{k}, Y^{k}\right\}\right)$ can be computed recursively from $p\left(x_{k-1}, c \mid\left\{Z^{k-1}, Y^{k-1}\right\}\right)$ within the framework of two steps - prediction and measurement update [2,4].

The predicted state-class pdf $p\left(x_{k}, c \mid\left\{Z^{k-1}, Y^{k-1}\right\}\right)$ at time $k$ is given by

$$
\begin{aligned}
p\left(x_{k}, c \mid\left\{Z^{k-1}, Y^{k-1}\right\}\right) & = \\
& \int_{x_{k-1}} p\left(x_{k} \mid x_{k-1}, c\right) p\left(x_{k-1}, c \mid\left\{Z^{k-1}, Y^{k-1}\right\}\right) d x_{k-1},
\end{aligned}
$$

where the conditional state prediction pdf $p\left(x_{k} \mid x_{k-1}, c,\left\{Z^{k-1}, Y^{k-1}\right\}\right)$ is obtained from the state transition equation (1).

The conditional pdf of the measurements $p\left(\left\{z_{k}, y_{k}\right\} \mid x_{k}, c\right)=\lambda_{\left\{x_{k}, c\right\}}\left(\left\{z_{k}, y_{k}\right\}\right)$ is usually known. This is the likelihood $\lambda_{\left\{x_{k}, c\right\}}\left(\left\{z_{k}, y_{k}\right\}\right)$ of the joint state and feature. When the measurements $\left\{z_{k}, y_{k}\right\}$ arrive, the update step can be completed

$$
p\left(x_{k}, c \mid\left\{Z^{k}, Y^{k}\right\}\right)=\frac{1}{\bar{d}_{k}} \lambda_{\left\{x_{k}, c\right\}}\left(\left\{z_{k}, y_{k}\right\}\right) p\left(x_{k}, c \mid\left\{Z^{k-1}, Y^{k-1}\right\}\right),
$$

where $\bar{d}_{k}=\sum_{c \in C} \int_{x_{k}} \lambda_{\left\{x_{k}, c\right\}}\left(\left\{z_{k}, y_{k}\right\}\right) p\left(x_{k}, c \mid\left\{Z^{k-1}, Y^{k-1}\right\}\right) d x_{k}$ is a normalizing constant. The recursion (4)-(5) begins with the prior density $P\left\{x_{0}, c\right\}, x_{0} \in$ $\mathbb{R}^{n_{x}}, c \in C$, which is assumed known. Then using Bayes' theorem, the target classification probability is calculated by the equation

$$
P\left(c \mid\left\{Z^{k}, Y^{k}\right\}\right)=\frac{p\left(\left\{z_{k}, y_{k}\right\} \mid c,\left\{Z^{k-1}, Y^{k-1}\right\}\right) P\left(c \mid\left\{Z^{k-1}, Y^{k-1}\right\}\right)}{\sum_{c \in C} p\left(\left\{z_{k}, y_{k}\right\} \mid c,\left\{Z^{k-1}, Y^{k-1}\right\}\right) P\left(c \mid\left\{Z^{k-1}, Y^{k-1}\right\}\right)}
$$

with an initial prior target classification probability $P_{0}(c)$, and $\sum_{c \in C} P_{0}(c)=1$. The class-dependent state estimate $\hat{x}_{k}^{c}, c \in C$ takes part in the calculation of the combined state estimate $\hat{x}_{k}$

$$
\hat{x}_{k}^{c}=\int_{x_{k}} x_{k} p\left(x_{k}, c \mid\left\{Z^{k}, Y^{k}\right\}\right) d x_{k}, \quad \hat{x}_{k}=\sum_{c \in C} \hat{x}_{k}^{c} P\left(c \mid\left\{Z^{k}, Y^{k}\right\}\right) .
$$


It can be seen from (4)-(6) that the estimates, needed for each class, can be calculated independently from the other classes. Therefore, the JTC task can be accomplished by the simultaneous work of $M$ independent filters [2.4]. The scheme of the particle filter bank, implemented in the present paper is described in Section 3.

It should be noted that because in our case we don't have feature measurements, the set $\left\{Y^{k}\right\}$ is replaced in the particle filter by the speed estimates from the $M$ classes. Together with a speed envelope which form is given in Section 3. they form a virtual "feature measurement".

\section{Particle Filter for Maneuvering Target Tracking and Classification}

Maneuvering target model. The two-dimensional target dynamics is given by

$$
x_{k}=F x_{k-1}+G\left[u_{k}+w_{k}\right], k=1,2, \ldots
$$

where the state vector $x=(x, \dot{x}, y, \dot{y})^{\prime}$ contains target positions and velocities in the horizontal $(O x y)$ Cartesian coordinate frame. The control input vector $u=\left(a_{x}, a_{y}\right)^{\prime}$ includes target accelerations along $x$ and $y$ coordinates. The process noise $w=\left(w_{x}, w_{y}\right)^{\prime}$ models perturbations in the accelerations. The transition matrices $F$ and $G$ are [9]

$$
F=\operatorname{diag}\left[F_{1}, F_{1}\right], \text { for } F_{1}=\left[\begin{array}{ll}
1 & T \\
0 & 1
\end{array}\right] ; \quad G=\operatorname{diag}\left[g_{1}, g_{1}\right], \text { for } g_{1}=\left[\frac{T^{2}}{2} T\right]^{\prime},
$$

where $T$ is the sampling interval. The target is assumed to belong to one of two classes $(M=2)$, representing either a lower speed commercial aircraft with limited maneuvering capability $\left(c_{1}\right)$ or a highly maneuvering military aircraft $\left(c_{2}\right)$ [7]. The flight envelope information comprises speed and acceleration constrains, characterizing each class. The speed $v=\sqrt{\dot{x}^{2}+\dot{y}^{2}}$ of each class is limited respectively to the interval:

$$
\left\{c_{1}: v \in(100,300)\right\}[\mathrm{m} / \mathrm{s}] \text { and }\left\{c_{2}: v \in(150,650)\right\}[\mathrm{m} / \mathrm{s}] .
$$

The range of the speed overlap section is $[150,300]$. The control inputs are restricted to the following sets of accelerations:

$$
\left\{c_{1}: u \in(0,+2 g,-2 g)\right\} \quad \text { and } \quad\left\{c_{2}: u \in(0,+5 g,-5 g)\right\},
$$

where $g\left[\mathrm{~m} / \mathrm{s}^{2}\right]$ is the gravity acceleration. The acceleration process $u_{k}$ is a Markov chain with five states $s\left(c_{1}\right)=s\left(c_{2}\right)=5$ :

$$
\begin{aligned}
& \text { 1. } a_{x}=0, \quad a_{y}=0, \quad \text { 2. } a_{x}=A, \quad a_{y}=A, \quad 3 . \quad a_{x}=A, \quad a_{y}=-A \text {, } \\
& \text { 4. } a_{x}=-A, \quad a_{y}=A, \quad \text { 5. } \quad a_{x}=-A, \quad a_{y}=-A
\end{aligned}
$$

where $A=2 g$ stands for class $c_{1}$ target and $A=5 g$ refers to the class $c_{2}$. The two target types have equal transition probabilities $p_{i j}, i, j \in S: p_{i j}=0.7, i=j ; p_{1 j}=$ $0.15, j=2, \ldots, 5 ; p_{i j}=0.05, j \neq i, i, j=2, \ldots, 5$. The initial probabilities are selected as follows: $P_{0}(1)=0.6, P_{0}(2)=P_{0}(3)=P_{0}(4)=P_{0}(5)=0.1$. The standard deviations of the process noise $w \sim N\left(0, \operatorname{diag}\left(\sigma_{w x}^{2}, \sigma_{w y}^{2}\right)\right)$ are different for each mode and class:

$$
\begin{aligned}
& \left\{c_{1}: \sigma_{w}^{j}=5.5\left[\mathrm{~m} / \mathrm{s}^{2}\right], j=1, \ldots, 5\right\} \text { and } \\
& \left\{c_{2}: \sigma_{w}^{1}=7.5, \sigma_{w}^{j}=17.5\left[\mathrm{~m} / \mathrm{s}^{2}\right], j=2, \ldots, 5\right\}, \text { where }\left(\sigma_{w}=\sigma_{w x}=\sigma_{w y}\right) .
\end{aligned}
$$


Measurement model. The measurement model at time $\mathrm{k}$ is described by

$$
z_{k}=h\left(x_{k}\right)+v_{k}, \quad h(x)=\left(\sqrt{x^{2}+y^{2}}, \arctan \frac{x}{y}\right)^{\prime},
$$

where the measurement vector $z=(D, \beta)^{\prime}$ contains the distance to the target $D$ and bearing $\beta$, measured by the radar. The parameters of the measurement error vector $v \sim N(0, R), R=\operatorname{diag}\left(\sigma_{D}^{2}, \sigma_{\beta}^{2}\right)$ are as follows: $\sigma_{D}=100.0[\mathrm{~m}] ; \sigma_{\beta}=0.15[\mathrm{deg}]$. The sampling interval of $T=5[s]$ is chosen in the simulations below.

Speed constraints. Acceleration constraints are imposed on the filter operation by an appropriate choice of the control input in the target model. The speed constraints are enforced through speed likelihood functions. Using the speed envelope information, the speed likelihood functions are defined by the following relationships

$$
\begin{aligned}
& g_{1}\left(v_{k}^{c_{1}}\right)= \begin{cases}0.8, & \text { if } v_{k}^{c_{1}} \leq 100[\mathrm{~m} / \mathrm{s}] \\
0.8+\kappa_{1}\left(v_{k}^{c_{1}}-100\right), & \text { if }\left(100<v_{k}^{c_{1}} \leq 300\right) \text { for } \kappa_{1}=-0.7 / 200 \\
0.1, & \text { if } v_{k}^{c_{1}}>300[\mathrm{~m} / \mathrm{s}]\end{cases} \\
& g_{2}\left(v_{k}^{c_{2}}\right)= \begin{cases}0.1, & \text { if } v_{k}^{c_{1}} \leq 150[\mathrm{~m} / \mathrm{s}] \\
0.1+\kappa_{2}\left(v_{k}^{c_{2}}-150\right), & \text { if }\left(150<v_{k}^{c_{1}} \leq 650\right) \text { for } \kappa_{2}=0.85 / 500 \\
0.95 & \text { if } v_{k}^{c_{1}}>650[\mathrm{~m} / \mathrm{s}] .\end{cases}
\end{aligned}
$$

According to the problem formulation, presented in Section 2, two class-dependent filters are working in parallel. At time step $k$, each filter gives state estimate $\left\{\hat{x}_{k}^{c}, c=1,2\right\}$. Let us assume, that the estimated speed from the previous time step, $\left\{\hat{v}_{k-1}^{c}, c=1,2\right\}$, is a kind of "feature measurement". The likelihood $\lambda_{\left\{x_{k}, c\right\}}\left(\left\{z_{k}, y_{k}\right\}\right)$ is factorized [2]

$$
\lambda_{\left\{x_{k}, c\right\}}\left(\left\{z_{k}, y_{k}\right\}\right)=f_{x_{k}}\left(z_{k}\right) g_{c}\left(y_{k}^{c}\right),
$$

where $y_{k}^{c}=\hat{v}_{k-1}^{c}$. Practically, the normalized speed likelihoods represent estimated by the filters speed-based class probabilities. The posterior class probabilities are modified by this additional speed information at each time step $k$. The inclusion of the speed likelihoods is done after some "warming-up" interval, including filter initialization.

Particle Filter Algorithm. Consider the hybrid particle $\mathbf{x}=\{x, m, c\}$, containing all necessary state, mode and class information. Let $N_{c}$ the number of particles for class $c$. Then the detailed scheme of the proposed particle filter comprises the following steps:

1. Initialization, $\quad k=0$.

For class $c=1,2, \ldots, M$, set $P(c)=P_{0}(c)$

* For $j=1, \ldots, N_{c}$, sample

$\left\{x_{0}^{(j)} \sim p_{0}\left(x_{0}, c\right), m_{0}^{(j)} \sim\left\{P_{0}^{c}(m)\right\}_{m=1}^{s}(c), c^{(j)}=c\right\}$ and set $k=1$.

End for $c$ 
2. For $c=1, \ldots, M$ (possibly in parallel) execute

* Prediction step

For $j=1, \ldots, N_{c}$ generate samples

$m_{k}^{(j)} \sim\left\{p_{l m}^{c}\right\}_{m=1}^{s(c)}$ for $l=m_{k-1}^{(j)}$,

$w_{k} \sim N\left(0, Q\left(m_{k}^{(j)}, c\right)\right), \quad x_{k}^{(j)}=F x_{k-1}^{(j)}+G u_{k}\left(m_{k}^{(j)}, c\right)+G w_{k}$

* Measurement processing step : on receipt of a new measurement $\left\{z_{k}, y_{k}\right\}$ :

For $j=1, \ldots, N_{c}$ evaluate the weights $w_{k}^{(j)}=f\left(z_{k} \mid x_{k}^{(j)}\right) g_{c}\left(y_{k}^{c}\right)$,

where $f\left(z_{k} \mid x_{k}^{(j)}\right)=N\left(z_{k} ; h\left(x_{k}^{(j)}\right), R\right)$ and $g_{c}\left(y_{k}^{c}\right)=g_{c}\left(\hat{v}_{k-1}^{c(j)}\right)$;

calculate

$p\left(\left\{z_{k}, y_{k}\right\} \mid c,\left\{Z^{k-1}, Y^{k-1}\right\}\right)=\sum_{j=1}^{N_{c}} w_{k}^{(j)}$ and set $L(c)=\sum_{j=1}^{N_{c}} w_{k}^{(j)}$

* Selection step

normalize the weights $w_{k}^{(j)}=w_{k}^{(j)} / \sum_{j=1}^{N_{c}} w_{k}^{(j)}$

resample with replacement $N_{c}$ particles $\left(x_{k}^{(j)} ; j=1, \ldots, N_{c}\right)$

from the set $\left.\left(x_{k}^{(l)}\right) ; l=1, \ldots, N_{c}\right)$, according to the importance weights

* Compute updated state estimate and posterior model probability

$\hat{x}_{k}^{c}=\frac{1}{N_{c}} \sum_{j=1}^{N_{c}} x_{k}^{(j)} ; P^{c}\left(m_{k}=l\right)=\frac{\sum_{j=1}^{N_{c}} m_{k}^{(j)}=l}{\sum_{j=1}^{N_{c}} m_{k}^{(j)}}, l=1, \ldots, s(c)$

End for $c$

3. Output: Compute posterior class probabilities and combined output estimate

$$
\begin{aligned}
& P\left(c \mid\left\{Z^{k}, Y^{k}\right\}\right)=L(c) P\left(c \mid Z^{k-1}, Y^{k-1}\right) / \sum_{c=1}^{M} L(c) P\left(c \mid Z^{k-1}, Y^{k-1}\right) \\
& \hat{x}_{k}=\sum_{c=1}^{M} P\left(c \mid\left\{Z^{k}, Y^{k}\right\}\right) \hat{x}_{k}^{c}
\end{aligned}
$$

4. Set $k \longleftarrow k+1$ and go to step 2 .

\section{Simulation Results}

The performance of the implemented tracking filter is evaluated by simulations over a representative test trajectory, depicted in Fig. 1

Measures of performance. Root-Mean Squared Errors (RMSE) [9]: on position (both coordinates combined) and speed (magnitude of the velocity vector), average probability of correct discrete state identification, average probability of correct class identification and average time per update are used to evaluate the filter performance.

The results presented below are based on 100 Monte Carlo runs. The number of particles for each class is $N_{c}=3000$. The prior class probabilities are chosen as follows: $P_{0}(1)=$ $P_{0}(2)=0.5$. The parameters of base state vector initial distribution $x_{0} \sim N\left[x_{0} ; m_{0}, P_{0}\right]$ are selected as follows: $P_{0}=\operatorname{diag}\left\{150^{2}, 20.0^{2}, 150^{2}, 20.0^{2}\right\} ; m_{0}$ contains the exact initial target parameters.

Test trajectory. The target performs two coordinated turn maneuvers with normal accelerations $2 g$ and $-5 g$, respectively, within scan intervals $(16 \div 23)$ and $(35 \div 37)$. The 
selected speed value is $v=250[\mathrm{~m} / \mathrm{s}]$. Then a maneuver is performed with longitudinal acceleration of $1 g$ and normal acceleration of $2 g$ in the frame of 3 scans $(45 \div 47)$. The longitudinal acceleration increases the speed up to $v=400[\mathrm{~m} / \mathrm{s}]$. These maneuvers are followed by another two maneuvers (during scans $(48 \div 56)$ and $(63 \div 70)$ ) with normal accelerations of $2 g$ and $-2 g$, respectively. The speed values (from 250

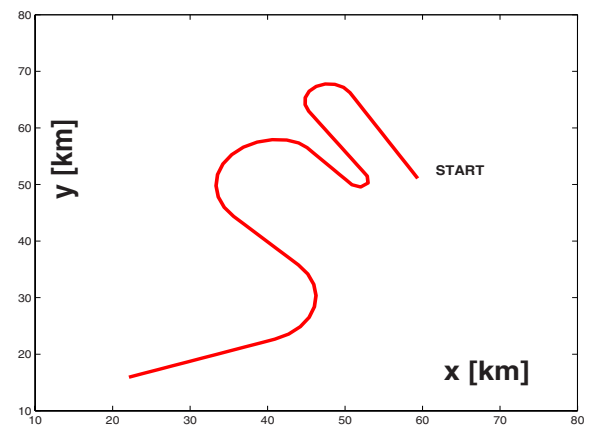

Fig. 1. Test trajectory (a) and

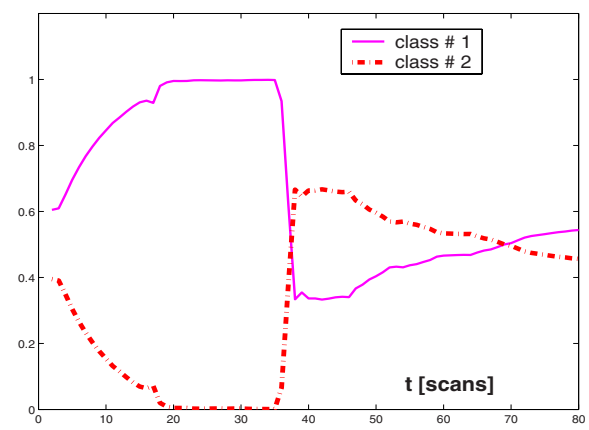

Fig. 2. Class probabilities without (a)

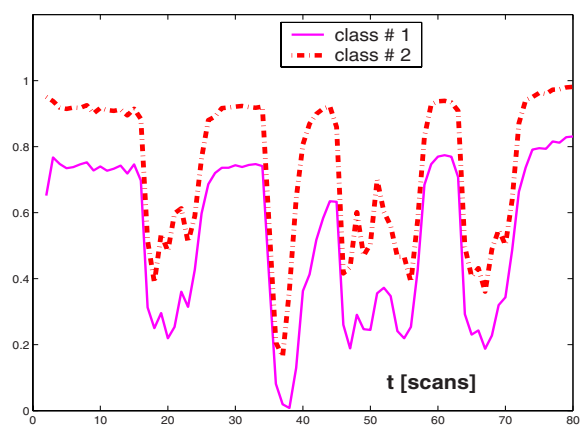

(b) Posterior probability of model 1

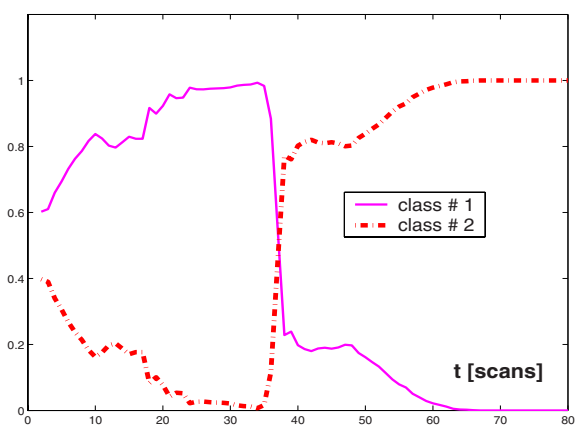

and (b) with speed constraints

to $400[\mathrm{~m} / \mathrm{s}]$ ) and the normal $5 \mathrm{~g}$ acceleration are typical for the second class target. After the $5 g$ maneuver, the filter correctly recognizes the real second class, but after the subsequent maneuvers of $2 g$, a tendency for misclassification is present (Fig.2(a)) in the filter without speed constraints. It is due to the fact, that the filtering system can "change its mind" regarding the class identification, if the second class target performs maneuvers, natural for the first class. The advantage of incorporation of speed constraints is illustrated in Fig. 2(b). According to the results from the RMSEs (Fig. 3) the developed particle filter with acceleration and speed constraints can reliably track maneuvering targets. The discrete (mode) states are determined correctly by the filter (Fig.1 b)). It 


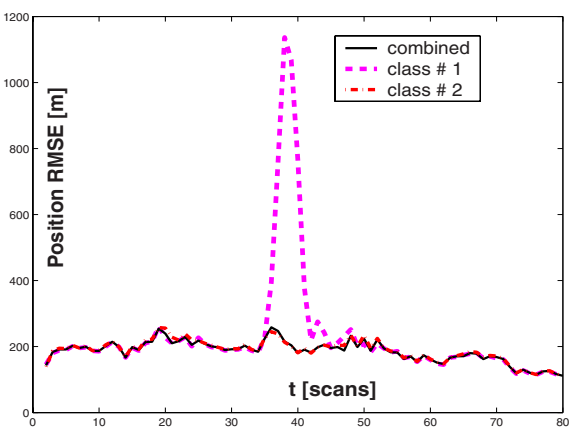

Fig. 3. Position RMSE [m] (a) and

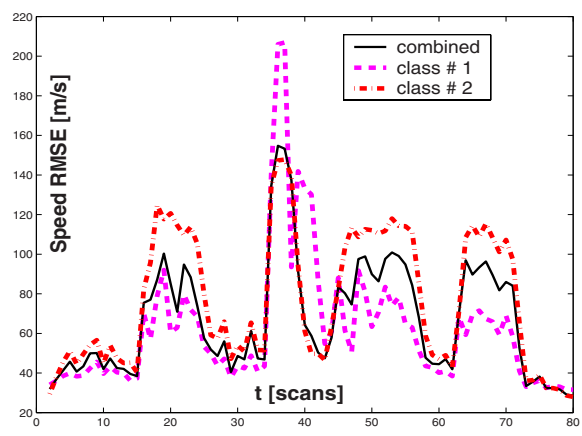

(b) Speed RMSE $[\mathrm{m} / \mathrm{s}]$

should be noted that the filter's computational complexity allows for an on-line processing. The average time per update, estimated in the MATLAB environment (on PC with AMD Athlon processor) is $1.52[s]$. It is less than the sampling interval of $5[s]$.

\section{Conclusions}

A Bayesian joint tracking and classification algorithm has been proposed recently in the work [2]. Based on this approach, a particle filter is developed in the present paper for maneuvering target tracking and classification. A bank of interacting multiple model class dependent particle filters is designed and implemented in the presence of speed and acceleration constraints. The acceleration constraints for each class are imposed by using different control inputs in the target model. The speed constraints are enforced by constructing class dependent speed likelihood functions. Speed likelihoods are calculated at each filtering step and assist in the process of classification.

The filter performance is analyzed by simulation over a typical $2-D$ target scenario. The results show a reliable tracking and correct target type classification.

\section{References}

1. Leung, H., Wu, J.: Bayesian and Dempster-Shafer target identification for radar surveillance. IEEE Trans. Aerospace and Electr. Systems, Vol. 36 (2000) 432-447

2. Gordon, N., Maskell, S., Kirubarajan, T.: Efficient particle filters for joint tracking and classification. Proc. SPIE Signal and Data Proc. of Small Targets, Vol. 4728, USA, (2002) 1-5

3. Herman, S., Moulin, P.: A Particle Filtering Appropach to FM-Band Passive Radar Tracking and Automatic Target Recognition. Proc. IEEE Aerospace Conf., Big Sky, Montana (2002)

4. Mallick, M., Maskell, S., Kirubarajan, T., Gordon, N.: Littoral Tracking Using Particle Filter. Proc. Fifth Int. Conf. Information Fusion, Annapolis, MD, USA (2002) 935-942

5. Challa, S., Bergman, N.: Target Tracking Incorporating Flight Envelope Information. Proc. Third International Conf. on Information Fusion, Paris, France (2000) ThC2-22-27

6. Tchamova, A., Semerdjiev, Tz., Dezert, J.: Estimation of Target Behaviour Tendencies using Dezert-Smarandache Theory. Proc. Sixth International Conf. on Information Fusion, Australia (2003) 1349-1356 
7. Challa, S., Pulford, G.: Joint target tracking and classification using radar and ESM sensors. IEEE Trans. Aerospace and Electr. Systems, Vol. 37 (2001) 1039-1055

8. Doucet,A., de Freitas, N., Gordon, N.(ed.): Sequential Monte Carlo Methods in Practice. Springer-Verlag, New York (2001)

9. Bar-Shalom, Y., Li, X.R.: Multitarget-Multisensor Tracking: Principles and Techniques. YBS Publishing (1995) 